\title{
Abstract: Generation of Annotated Brain Tumor MRIs with Tumor-induced Tissue Deformations for Training and Assessment of Neural Networks
}

\author{
Hristina Uzunova ${ }^{1}$, Jan Ehrhardt ${ }^{1}$, Heinz Handels ${ }^{1}$ \\ ${ }^{1}$ Institute of Medical Informatics, University of Lübeck \\ uzunova@imi.uni-luebeck.de
}

Machine learning methods, especially neural networks, have proven to excel at many image processing and analysis methods in the medical image domain. Yet, their success strongly relies on the availability of large training data sets with high quality ground truth annotations, e.g. expert segmentation of anatomical/pathological structures. Therefore, generating realistic synthetic data with ground truth labels has become crucial to boost the performance of neural networks. Most of the large publicly available datasets containing some type of pathologies are commonly designed for the segmentation (detection/ localization) of the particular pathological structure and thus only contain expert segmentations of the latter. On the other hand, datasets containing ground truth annotations of normal anatomy are usually generated from healthy populations. This leads to a lack of ground truth annotation to evaluate the accuracy of standard algorithms on pathological data, and a lack of data to train algorithms that target anatomical structures in pathological data. In this work, we propose a method for the generation of realistic pathological data with ground truth labels of both anatomical and pathological structures [1]. This is achieved by a GAN-based domain translation approach, that retains the topology of a healthy source domain, whereas the appearance of a target pathological domain is recreated. This way, the anatomic annotations of the source domain, can be directly applied to the generated images. Our method also includes an explicit pathology injection with available ground truth segmentations. Furthermore, we propose a novel inverse probabilistic approach to simulate tumor-induced deformations of the surrounding tissue. For our experiment brain tumor MRIs are generated and used for training and evaluation of segmentation and registration neural networks, proving the feasibility of our approach.

\section{References}

1. Uzunova H, Ehrhardt J, Handels H. Generation of annotated brain tumor MRIs with tumor-induced tissue deformations for training and assessment of neural networks. Proc MICCAI. 2020; p. 501-511. 